\title{
Hollow-Core Bragg Fiber for Bio-Sensing Applications
}

\author{
K. MileńKO ${ }^{a, *}$, D.J.J. Hu ${ }^{b}$, P.P. Shum ${ }^{c}$ And T.R. Woliński ${ }^{a}$ \\ ${ }^{a}$ Faculty of Physics, Warsaw University of Technology, Koszykowa 75, 00-662 Warszawa, Poland \\ ${ }^{b}$ Institute for Infocomm Research, Agency for Science, Technology and Research (A*STAR), Singapore \\ ${ }^{c}$ School of Electrical and Electronic Engineering, Nanyang Technological University, Singapore \\ Theoretical analysis of propagation properties in a hollow-core Bragg fiber for bio-sensing applications has \\ been demonstrated. Based on the Bragg fiber we propose a resonant sensor that operates on changes in refractive \\ index of aqueous solution placed inside the hollow core. By using the transfer matrix method we analyzed the \\ confinement loss of the $\mathrm{TE}_{01}$ mode in the hollow-core Bragg fiber. We have shown the influence of the fiber \\ geometry on the changes in the confinement loss. Spectral sensitivity and resolution of the sensor are also presented.
}

PACS numbers: 87.85.fk, 42.81.Pa, 42.81.-i, 42.79.Dj

\section{Introduction}

Photonic crystal fibers (PCFs) are special optical fibers with a periodic structure. Propagation of light in a PCF depends on the geometry and structural parameters of the fiber. Light can be guided due to total internal refraction (TIR) when the refractive index of the core is higher than the cladding or by the photonic band gap (PBG) effect. In the case of PBG light is propagating in a core with the refractive index that is smaller than the refractive index of the cladding thanks to the Bragg reflection. A very first photonic band gap optical fiber presented by Yeh in 1978 was the Bragg fiber [1-3]. Cladding of the Bragg fiber is composed of concentric circular layers with alternately higher and lower refractive indices. Recently, the Bragg fibers gain more attentions because of new techniques of their fabrication [4-6].

We present a hollow-core Bragg fiber that can be used in bio-sensing applications. Fiber-optic biosensors are devices which use an optical field to measure biomaterial samples such as cells, proteins and DNA. The advantages of the optical fiber sensors are associated with their immunity to electromagnetic interference, light weight, small size, high sensitivity, large bandwidth, and high transmission speed. They may have many new and important applications like drug discovery, medical diagnostic, environmental monitoring, and protection against bioterrorism. Hollow-core Bragg fibers show utility in bio-sensing because they give access for a sample to regions with strong overlap with an optical field without taking off fiber cladding and its coating. This makes the sensor more solid. Optical properties of the Bragg fibers are determined by the numbers, thickness and refractive index of layers and these properties can be easily designed by fiber geometric simulations. The hollow-core fiber can be infiltrated with liquid or gaseous sample and the sample volume needed for measurements is small. Aqueous

\footnotetext{
* corresponding author; e-mail: karolina.milenko@gmail.com
}

solution infiltrated into the core introduces changes in properties of the propagating light. This feature allows investigation of the solution refractive index by studying shift of the band gaps peaks.

In the paper we analyze the confinement loss of the hollow-core Bragg fiber. In the second section of the paper we describe parameters of the examined optical fiber. Section three shows how modifications in geometric parameters of the Bragg fiber influence changes in the confinement loss. We present relationship between minimal value of the confinement loss for different core radius, number of layer pairs and refractive index of the cladding. The possibility to control the position of the band gaps peak is also presented. Finally, we introduce spectral sensitivity and sensor resolution.

\section{Parameters of the proposed Bragg fiber}

We have considered a circular hollow-core Bragg fiber with a cladding composed of the layers with different refractive indices (Fig. 1). The analyzed fiber was characterized by the following parameters: number of layer pairs $N=4$, the core radius $R=10 \mu \mathrm{m}$, refractive indices: core $n_{0}=1$, first layer $n_{1}=1.8$, second layer $n_{2}=1.5$, the period $\Lambda=1.2 \mu \mathrm{m}$.
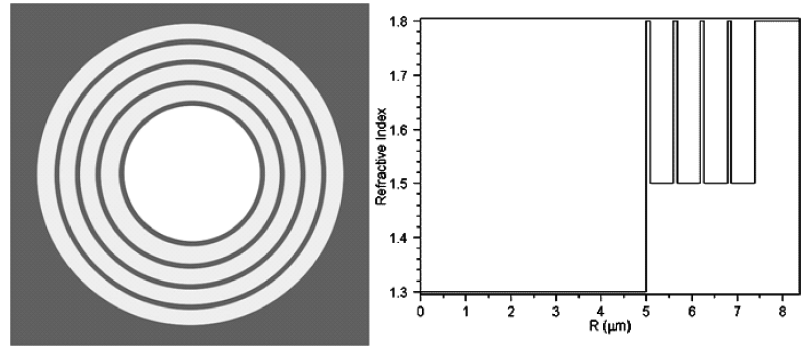

Fig. 1. Scheme of the Bragg fiber filled with aqueous solution: (a) the cross-section, (b) refractive index profile.

To analyze propagation properties of the Bragg fiber we employed the transfer matrix method (TMM) using 


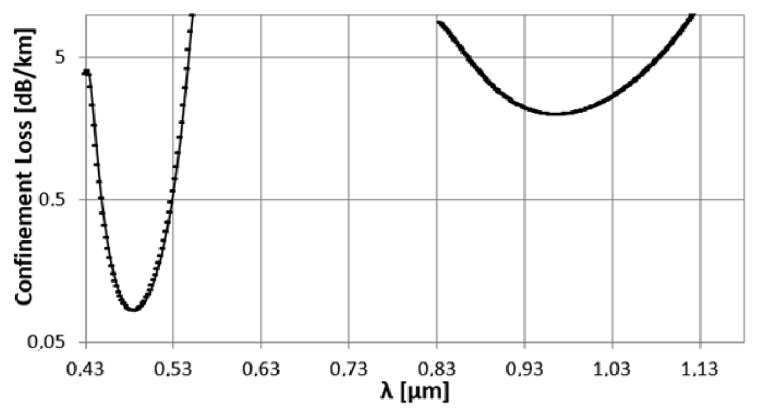

Fig. 2. Confinement loss of the $\mathrm{TE}_{01}$ mode.

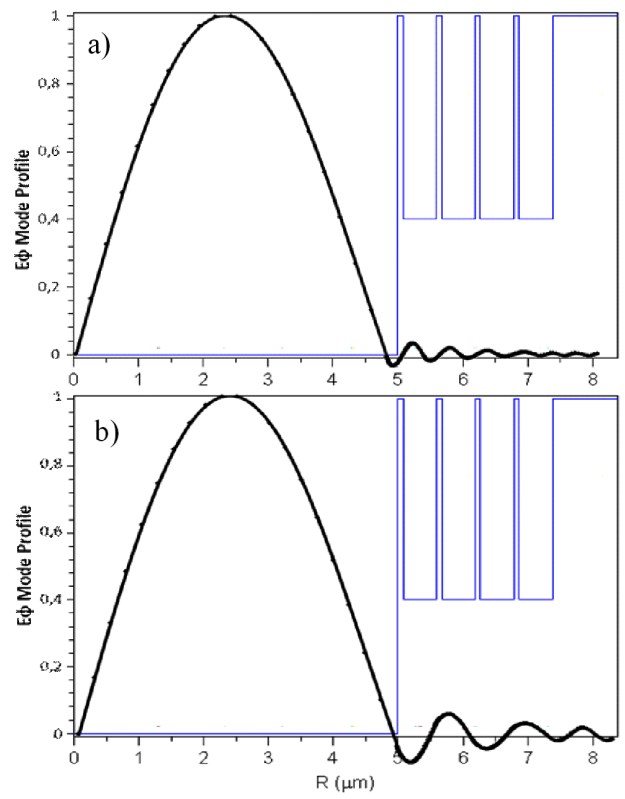

Fig. 3. Electric field of the $\mathrm{TE}_{01}$ mode at wavelength (a) $\lambda=0.484 \mu \mathrm{m}$, (b) $\lambda=0.966 \mu \mathrm{m}$.

which the field components in every layer can be described as a linear combination of the Bessel functions [7]. TMM method gives an effective mode index of the propagating light. For the TMM simulations we used RSOFT software.

From the imaginary part of the effective mode index we calculate confinement loss (CL) based on the equation [8]:

$$
\mathrm{CL}=\frac{40 \pi}{\lambda \ln 10} \operatorname{Im}\left(n_{\mathrm{eff}}\right) \times 10^{3}\left[\frac{\mathrm{dB}}{\mathrm{km}}\right] .
$$

The confinement loss of the $\mathrm{TE}_{01}$ mode for the proposed Bragg fiber parameters is showed in Fig. 2.

The minimum confinement loss for $\mathrm{TE}_{01}$ mode in the fundamental gap is $\mathrm{CL}_{\min }=2.0 \mathrm{~dB} / \mathrm{km}$ at wavelength $\lambda=0.966 \mu \mathrm{m}$ and in second order band gap $\mathrm{CL}_{\min }=$ $0.8 \times 10^{-1} \mathrm{~dB} / \mathrm{km}$ at $\lambda=0.484 \mu \mathrm{m}$. The confinement loss in second order band gap is one magnitude lower than the confinement loss in the fundamental band gap. This is consistent with the analysis result reported in [9]. The profile of the $\mathrm{TE}_{01}$ mode for the minimum confinement loss is presented in Fig. 3.

\section{Confinement loss analysis}

Confinement loss of the Bragg fiber can be easily changed by modifications in the geometric parameters of the fiber. Basing on this property we investigated how to reduce it in a most efficient way and we analyzed the confinement loss of $\mathrm{TE}_{01}$ mode for the second order band gap after introducing the geometric changes.

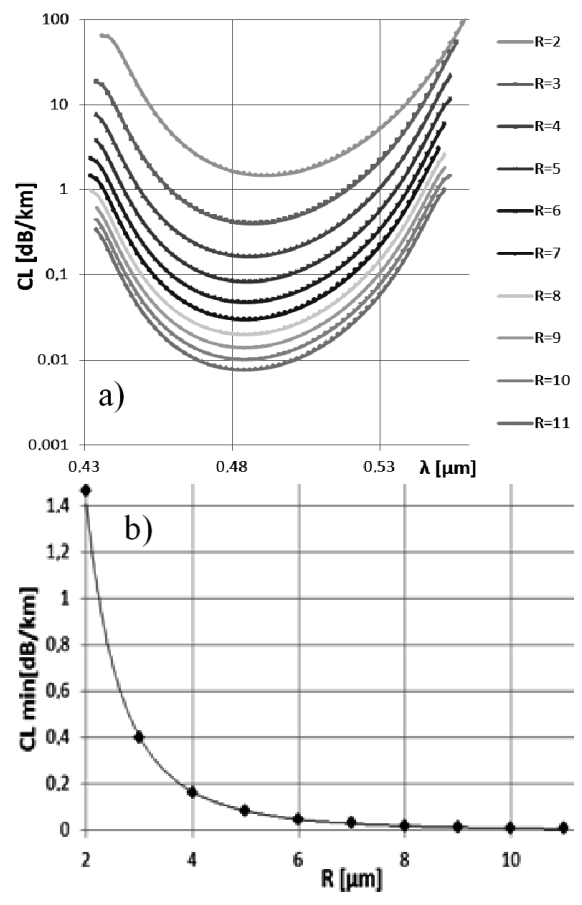

Fig. 4. Confinement loss of the $\mathrm{TE}_{01}$ mode for different core radius, (b) $\mathrm{CL}_{\min }$ for different core radius.

First we took into account different core radius $R$ (Fig. 4a). We calculated the confinement loss for the core radius in $2-10 \mu \mathrm{m}$ range. Figure $4 \mathrm{~b}$ shows a decrease in the confinement loss with the increasing core size between 2 and $6 \mu \mathrm{m}$. The minimal confinement loss is reducing rapidly for $R=2 \mu \mathrm{m}$ : $\mathrm{CL}_{\min }=1.46 \mathrm{~dB} / \mathrm{km}$ and for $R=6 \mu \mathrm{m}$ : $\mathrm{CL}_{\min }=0.05 \mathrm{~dB} / \mathrm{km}$. For larger cores, changes in the confinement loss are smaller. For the core radius $R=11 \mu \mathrm{m}$ we obtained minimum confinement loss $\mathrm{CL}_{\min }=0.01 \mathrm{~dB} / \mathrm{km}$. These results show that increasing the core radius above some values brings little benefits.

Since the number of layer pairs $N$ influences losses, we investigate confinement loss for number of layers from $N=3$ to $N=10$ (Fig. $5 \mathrm{a}$ ).

Results in Fig. 5b demonstrate that the minimum confinement loss is an exponential function of the layers number. After increasing the number of layers to $N>8$ the minimum confinement loss is diminishing very slowly. This property is important in case of fiber fabrication, because making a fiber with large number of layers is more difficult.

Comparing the confinement loss for different core radiuses and numbers of layers we can see that the function 

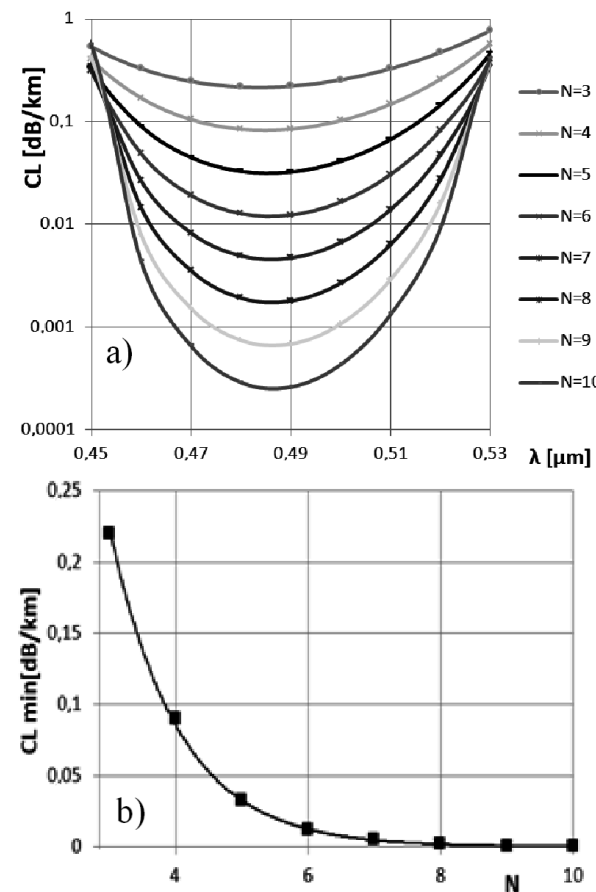

Fig. 5. (a) Confinement loss of the TE mode for different number of layers, (b) $\mathrm{CL}_{\min }$ for different number of layers.
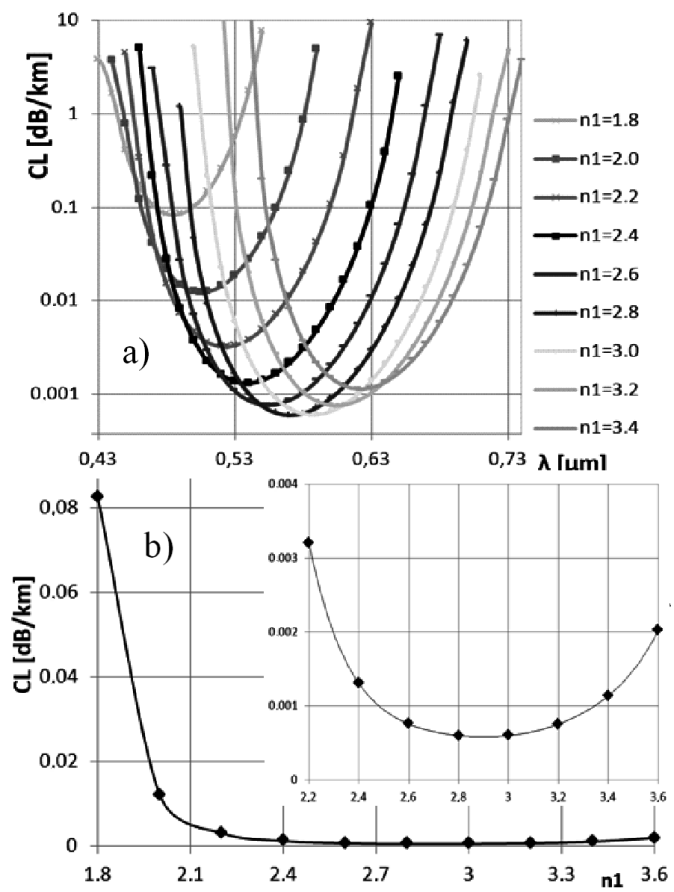

Fig. 6. Confinement loss of the $\mathrm{TE}_{01}$ mode for different refractive index of the layer $n_{1}$, (b) $\mathrm{CL}_{\min }$ for different refractive index of the layer $n_{1}$.
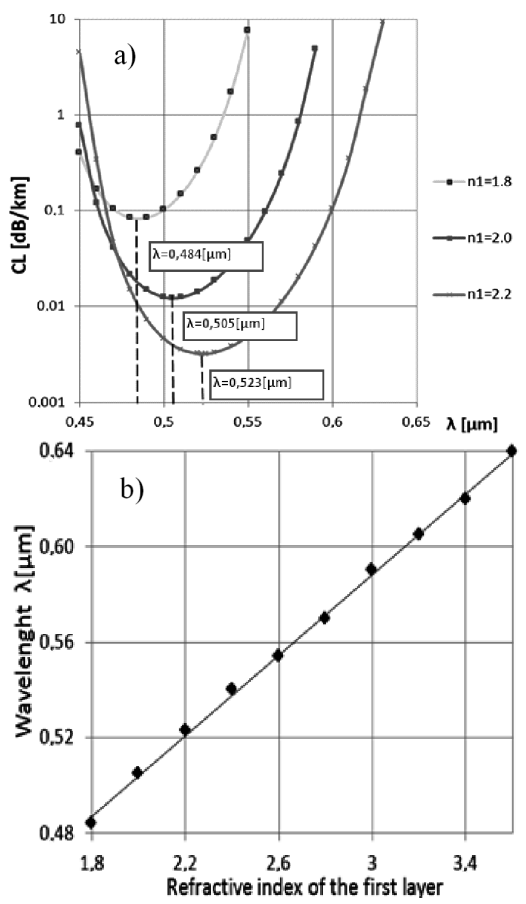

Fig. 7. Confinement loss of the TE mode for different refractive index of the layer $n_{1}$, (b) wavelength in function of the first layer refractive index.

of the minimum confinement loss is similar but Fig. 4a and Fig. 5a show that when we increase the core radius the whole band gap moves down and for the bigger number of layers the gap lengthens.

When we increase the refractive index of the first layer, the confinement loss is also changing. In the beginning, the confinement loss is decreasing rapidly and when the refractive index of the first layer reaches 2.9 , the confinement loss is starting to increase (Fig. 6b).

Enlarging the difference between refractive indexes of the cladding layers causes the band gap shift into the higher wavelengths (Fig. 6a). In Fig. 7a we present the confinement loss of the $\mathrm{TE}_{01}$ mode for refractive indices of the first layer $n_{1}=1.8 ; 2.0 ; 2.2$. From this figure we can see not only the band gap shifting, but also that it is expanding. The shift of the band gap peak position is a linear function of the refractive index of the first layer (Fig. 7a).

By changing the refractive index of the cladding we manipulate the PBG peak position which results in a possibility to choose the suitable wavelength with minimal confinement loss.

Summarizing, the above: changes in the core radius allow us to decrease the confinement loss maintaining the wave length properties, changing the number of layers lowers the band gap width of the studied fiber and rising the first layers refractive index shifts the band gap towards higher wavelengths. Those properties together allow us to determine the exact properties of a designed fiber according to our needs. 
4. Spectral sensitivity of the sensor

One of the most popular absorption based sensors methodology is spectral sensing [10]. Spectral sensing is based on detection of PGB shift. The position of the absorption peak changes in the presence of a measured solution. Spectral sensitivity can be calculated from the equation

$$
S_{\lambda}=\frac{\partial \lambda(\delta)}{\left.\partial \delta\right|_{\delta=0}},
$$

where $\delta$ is a value of the measured solution.

The sensor resolution described as the minimum value of the measured solution that can be detected is

$$
\delta_{\min }=\frac{\left(\Delta \lambda_{\mathrm{p}}\right)_{\min }}{S_{\lambda}},
$$

where the $\left(\Delta \lambda_{\mathrm{p}}\right)_{\min }$ is the minimal spectral shift in the peak position.

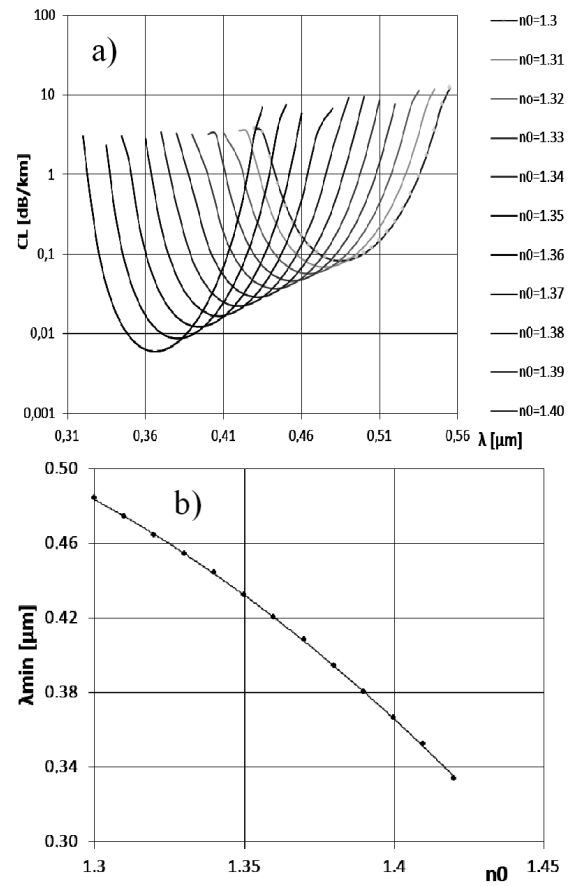

Fig. 8. Confinement loss for different solution refractive index, (b) the peak position as a function of core refractive index for a second order band gap.

To investigate sensitivity of the proposed fiber we calculated confinement loss for different refractive index of the core. We considered the refractive index of the aqueous samples in the range of $1.3 \div 1.4$. Figure 8 a shows the confinement loss of the $\mathrm{TE}_{01}$ mode in function of wavelength for different core refractive indices in second-order PBG. When we increase the core refractive index the position of PBG shifts into the lower wavelengths.

For the sensitivity calculation we plotted peak position $\left(\Delta \lambda_{\mathrm{p}}\right)_{\text {min }}$ in function of the measured value: the core refractive index (Fig. 8b). By using Eqs. (2) and (3) we could calculate sensitivity and resolution of the proposed sensor. As a result, the sensor sensitivity in the second order band gap is $S_{\lambda}=870 \mathrm{~nm} / \mathrm{RIU}$ (RIU - refractive index unit) whereas the resolution is $1 \times 10^{-4}$.

\section{Conclusion}

We have introduced a low-loss Bragg fiber that can be used for bio-sensing applications. The confinement loss of the $\mathrm{TE}_{01}$ mode was theoretically analyzed. We have calculated the confinement loss for different core radius, numbers of layers and refractive index of the cladding. We demonstrated possibility to shift position of the band gap peak. By testing different refractive indices of the hollow-core filling we calculated sensitivity and resolution of the sensor. These results would be helpful to design a highly sensitive biosensor based on the hollow core Bragg fiber.

\section{Acknowledgments}

This work was supported by Nanyang Technological University and by the Foundation for Polish Science in the frame of "Master Program".

\section{References}

[1] P. Yeh, A. Yariv, E. Marom, J. Opt. Soc. Am. 68, 1196 (1978).

[2] T. Pustelny, M. Grabka, Acta Phys. Pol. A 114, A-113 (2008).

[3] T. Pustelny, M. Grabka, Acta Phys. Pol. A 116, 385 (2009).

[4] E. Pone, C. Dubois, N. Guo, Y. Gao, A. Dupuis, F. Boismenu, S. Lacroix, M. Skorobogatiy, Opt. Exp. 14, 5838 (2006).

[5] D. Budaszewski, A.W. Domański, Acta Phys. Pol. A 116, 285 (2009).

[6] A.W. Domański, P. Lesiak, K. Milenko, D. Budaszewski, M. Chychlowski, S. Ertman, M. Tefalska, T.R. Wolinski, K. Jedrzejewski, L. Lewandowski, W. Jasiewicz, J. Helsztynski, A. Boczkowska, Acta Phys. Pol. A 116, 294 (2009).

[7] T. Kawanishi, M. Izutsu, Opt. Exp. 7, 4321 (2000).

[8] S. Guo, S. Albin, R.S. Rogowski, Opt. Exp. 12, 198 (2004).

[9] K.J. Rowland, V. Shahraam Afshar, T.M. Monro, J. Lightwave Technol. 26, 13 (2008).

[10] M. Skorobogatiy, J. Sensors 11, 479 (2009). 